\title{
Ensemble Kalman Filter Predictor Bias Correction Method for Non-Gaussian Geological Facies Detection
}

\author{
Siavash Nejadi*, Japan J. Trivedi**, Juliana Leung*** \\ School of Mining and Petroleum Engineering, University of Alberta, Edmonton, Alberta, T6G 2 W2 Canada \\ * (Tel: 780-492-5207; e-mail: nejadi@ualberta.ca) \\ ** (Tel: 780-492-1247; e-mail: jtrivedi@ualberta.ca) \\ ***(Tel: 780-492-3338; e-mail: juliana2@ualberta.ca)
}

\begin{abstract}
:
The Ensemble Kalman Filter (EnKF) is a Monte-Carlo based technique for assisted history matching and real time updating of reservoir models. However, it often fails to detect facies boundaries and proportions as the facies distributions are non-Gaussian, while geologic data for reservoir modeling is usually insufficient. It is convenient to represent distinct facies with non-Gaussian categorical indicators; we implemented discrete cosine transform (DCT) to parameterize the facies indicators into coefficients of the retained cosine basis functions that are Gaussian. For highly complex and heterogeneous models, though observed data were matched, it failed to reproduce realistic facies distribution corresponding to reference variogram and facies proportion.

In this paper we propose a new ensemble filtering method in-between of EnKF and PF, where EnKF as predictor combines the advantages of accurate large updates with small ensembles and corrector for nonGaussian distributions followed by EnKF again for analysis step. Correction is performed by regenerating new realizations using a new pilot point method. The ensemble members that are more consistent with the early production history and the available geological information are considered as high weight particles and used for the applications.

Combination of DCT-EnKF and regenerating new realizations using the new pilot point method demonstrates reasonable improvement and reduction of uncertainty in facies detection. Incorporating the new step in the procedure assists the filter to honor the reference distribution and experimental variogram during the history matching process and presents an important potential in improved characterization of complex reservoirs.
\end{abstract}

Keywords: Ensemble Kalman Filter, EnKF, Facies Detection, History Matching, Particle Filter, Predictor Corrector

\section{INTRODUCTION}

The Ensemble Kalman Filter (EnKF) is a Monte-Carlo based technique for data assimilation and has been introduced to the petroleum engineering industry by Lorentzen et al. (2001) as a promising approach for solving high-dimensional history matching problems (Aanonsen et al. 2009). The EnKF implements an ensemble of model states and approximates the covariance matrices sequentially in time as new observations become available. Each member of the ensemble is updated using an ensemble approximation to the Kalman gain, which is approximated from the mean and covariance of the prior joint probability density function (pdf). The updated ensemble provides an empirical estimate of the posterior joint probability distribution. Kalman filter performs well when there is a linear relationship between states variables, model parameters, and the data. The underlying assumption is that the prior joint pdf is Gaussian when computing the updates and EnKF will not converge to the correct distribution, identified by geological studies, if the prior joint pdf has non-Gaussian contributions. In other words the estimated posterior pdf is not consistent with the prior distribution (Zafari and Reynolds 2007).

This assumption of Gaussianity implies that the conventional EnKF method must be modified such that it can be applied for models whose petrophysical properties are not characterized by multivariate Gaussian distributions (Evensen 2007, Aanonsen et al. 2009). For instance, facies modelling is a crucial component of geostatistical reservoir characterization which facilitates construction of models for complex reservoirs. Facies are commonly represented by indicators, which are intrinsically non-Gaussian. Different approaches can be found in the literature to model nonGaussian parameters, namely, Truncated Pluri-Gaussian (Liu and Oliver 2005, Agbalaka and Oliver 2008), Level Set Method (Moreno and Aanonsen 2011, Lorentzen et al. 2011a, 2011b), Gaussian Mixture Models (Dovera and Della Rossa 2010) and Discrete Cosine Transform (Jafarpour et al. 2008).

Though the aforementioned techniques have shown some success for facies detection in synthetic models, there are some common shortcomings in these techniques. Firstly except the truncated pluri-Gaussian and level set technique, 
these methods have not been tested or are not applicable for reservoirs with more than two distinct facies. Secondly these methods may not capture the information regarding model parameters that is inferred from static geological data. It is important to condition models to reference geologic information and dynamic flow data in reservoir characterization. In many cases, static data (e.g. conceptual models, log, core, seismic interpretations, and statistics from similar fields) are available for reservoir modelling. Therefore, characterization of a robust description of geological features such as facies distributions (proportions and spatial patterns) and channel properties (e.g. orientation, widths and amplitudes) should incorporate all the static information and honor the reference statistics (e.g. histogram and semi-variogram or covariance) inferred from the reference geologic information.

While predicting model parameters using EnKF, the initial ensemble is designed to account for all uncertainties in the reservoir model. Major portion of the ensemble uncertainty will be diminished after the few EnKF update steps (Jafarpour et al. 2011) and realizations with initial poor estimates, undertake major changes to match early production history. As a result of this early update, spatial relation of the properties is destructed and the model parameter variogram will not follow the geological properties of the formation, based on which the initial ensemble was generated. The calculated experimental variogram along different azimuths was not in accordance with reference benchmark variogram and the short scale variability was systematically larger for all the updated realizations. Furthermore, after even more update steps EnKF transforms the initials multimodal distribution to a more Gaussian distribution (Zafari and Reynolds 2007) and the first order statistical features of the data (e.g. histogram) will be missed as well.

The motivation of this paper is to present a modified approach to honor the reference statistics within the conventional framework of EnKF using parameterization of DCT and incorporating a re-sampling step. After initial EnKF update steps, a re-sampling procedure is implemented to generate an "improved" ensemble of initial model parameters. This idea of re-generating new realizations stems from the particle filtering approach, also used in data assimilation, where particles or ensemble members that have negligible weights should be discarded and replaced by highweight particles. In our implementation, "best" realizations representing minimal root mean square error compared to the observations and minimum variogram mismatch are selected. These realizations are more consistent with the early production history and the available geological information. In particular, a new approach with the use of pilot points is implemented to regenerate a new ensemble of initial model parameters after a few initial assimilation steps. The new ensemble incorporates the model updating information of the initial steps and consists of realizations that are more consistent with both the dynamic and static data. The new ensemble is subsequently updated using EnKF from the beginning. In this paper we begin our discussion with first introducing our DCT-EnKF methodology for updating facies indicators, followed by the corrector procedure to re-generate new set of realizations using the new Pilot Point method. Then we presented the results of the application of the methodologies in a synthetic reservoir model. At the end, the conclusion and implication drawn from the case studies are presented.

\section{METHODOLOGY}

EnKF updates each particle (ensemble member) directly, without re-weighting or re-sampling. A Gaussian approximation and linear update in EnKF introduce systematic bias because the true distributions can be significantly non-Gaussian (such as the cases for multi-facies models) and the relation between the observation and state may be nonlinear. The Particle filter (PF) is a Monte Carlo based algorithm which approximates the posterior distribution with weighted samples. The analysis distribution and forecast distribution can be very different for EnKF than PF.

In this paper, we propose a new ensemble filtering method inbetween of EnKF and PF, where EnKF as predictor combines the advantages of accurate large updates with relatively small ensembles and corrector for non-Gaussian distributions followed by EnKF again for analysis step. Conceptually, our approach is similar to predictor-corrector method where EnKF works as a predictor to move the ensemble members (state distribution) towards the correct region using measurement and observations, and then corrector adjust for non-Gaussian character of the distribution. However, in our approach we are not using weighted EnKF. Weights of ensembles are computed after predictor step (EnKF) using histogram and variogram mismatch. In corrector step, ensemble members with higher mismatch (lower weights) are discarded and ensembles with higher weights (lower mismatch) are subjected to a new pilot point scheme for generation of new members. It is important to note that the corrector step is performed after specific numbers of update steps, selected at the stage where the observations mismatch is low and stabilized. Furthermore, due to nonlinearity of reservoir simulation equations, the consistency between updated state vector and updated vector of model parameters occasionally breaks down. In such a case running the simulator from the beginning is necessary. Each ensemble member is advanced again by the model for analysis from the beginning using EnKF.

In this section, various components of the implementation are discussed in detail. Specific modifications to the conventional EnKF procedure are highlighted.

\subsection{Predictor - Ensemble Kalman Filter}

Initially the forecast model is separately applied to each ensemble member using a reservoir simulator.

$m_{k}^{p, j}=F\left(m_{k-1}^{a, j}\right), j=1, \ldots, N_{e}$

where superscript $p$ and $a$ denote the predicted and analyzed states, respectively, $k$ denotes the time step in which data are assimilated and $N_{e}$ is the number of realizations.

Discrete Cosine Transform (DCT), a Fourier-based transform, is applied to decompose the spatial distribution of 
model parameters (in this case facies indicators) into coefficients of the retained cosine basis functions. DCT is performed separately on each realization of the initial ensemble and certain number of basis functions and the corresponding coefficients are retained. It is noted that the total number of basis functions $(r)$ can be much less than the total number of model parameters which is the number of grid blocks in the model. The DCT coefficients are incorporated into the state vector instead of the model parameters $(m)$. This model state is represented by a weighted ensemble, where, here the weights are same for each realization $\left(w_{i}=1 / N_{e}, i=1,2, \ldots, N_{e}\right)$. EnKF procedure is used to update model parameters $(m)$ and state variables $(u)$.

$y_{k}=\left\{\begin{array}{lll}m_{k} & u_{k} & d_{k}\end{array}\right\}^{T}$

where $d$ denotes the observations.

After the EnKF update step, inverse DCT transform is used to convert back the updated DCT coefficients into facies indicator values. These updated indicators are further used in the subsequent forecast and update steps as new observations become available.

\subsection{Corrector - Ensemble Kalman Bias Correction using Re-sampling and Ensemble Regeneration}

The corrector step is applied after major change in the model in predictor step for bias correction. The role of corrector is to assign importance weights by mismatch calculation (RMSE) of observations. This does not change the ensemble or the weights. The members with larger weights are the only contributors of non-Gaussian nature. Therefore, similar to sequential importance re-sampling in $\mathrm{PF}$, new ensemble members are generated to replace the members with low weights. Hence, the variance of the importance weights doesn't increases inevitably (avoiding degeneracy phenomenon) and stability is maintained. The generations of new ensemble members are done using pilot point method. This newly constructed proposal ensemble has all weights equal and is again forwarded in time by EnKF from the beginning. It should be noted that information from the previous update steps are retained only at the pilot points, and propagated to other locations through conditional simulation. This scheme allows us to condition a new set of initial ensemble using the dynamic information, in a way similar to incorporating additional hard data observed at the well locations. i.e. dynamic data has become part of the initial data $\left(d_{0}\right)$ after each re-sampling step. Since the re-sampling algorithm is applied only at the re-sampling step (e.g., once in our case study) the dynamic information would not be used twice within a single update step, as in other weighted EnKF formulations, hence the prior and likelihood probabilities (Eq. 3) remain independent during each update step.

$$
p\left(\vec{m}_{k} \mid \vec{d}_{k}\right) \quad \alpha \quad p\left(d_{o b s, k} \mid \vec{m}_{k}, \vec{d}_{k-1}\right) p\left(\vec{m}_{k} \mid \vec{d}_{k-1}\right)
$$

\subsection{Pilot Point}

The use of pilot points has been proposed by de Marsily et al. (1984) as a re-parameterization technique in traditional history matching to reduce the size of the parameter space during updating. The parameter estimations at selected locations in the reservoir named "pilot points" are updated in the history matching procedure. The objective function is constructed by comparing the data mismatch at the locations of the pilot point. Model parameters at pilot points are perturbed, and the changes made at the pilot points are propagated to other locations by performing stochastic simulation using model values at pilot points as part of the conditioning data, together with geostatistical parameters (e.g. histogram and semi-variogram models), to preserve the spatial correlation between estimation locations. Taking advantage of this concept, predicted facies values at pilot points were used for re-generating the new realizations. Facies values at the pilot points were derived from the selected updated realizations to be further implemented as the conditioning hard data for the stochastic simulation. Sequential Indicator Simulation (SIS) was used for stochastic simulation that included geostatistical parameters of the field.

A practical consideration is the specification of the location and number of the pilot points. RamaRoa et al. (1995) proposed placing the pilot points in high sensitivity zones where they have the highest potential to reduce the objective function and correctly locate the heterogeneities. Unfortunately, the number of pilot points is inherently empirical.

Number of pilot points: The number of pilot points should be selected depending on the nature of the problem. Factors such as reservoir model, well pattern, production mechanism, reservoir heterogeneity and other parameters should be taken into consideration to determine the number of pilot points. The number of pilot points may be optimized such that they are large enough to capture the adjustments made to model parameters during the previous updating steps in the regenerated ensemble while being small enough to ensure that the entire model parameter space is sufficiently sampled. The numbers of pilot points in our case studies are 10 percent of the total number of grid blocks.

Location of pilot points: In contrast with other applications of pilot point methods where their locations were specified a priori, we have selected the pilot point locations after updating the model parameters via EnKF. Members with larger weights were identified and used to calculate the probability density function (pdf) of facies indicators for all grid blocks. The pilot points were selected at grid blocks which have the highest probability of the occurrence of a specific facies. Probability of the occurrence of a specific facies at any grid block would be high (close to one), if the same facies type exists at that grid block in different realizations. The idea is to select/ identify locations where there is the least uncertainty in the model parameter (portions of the solution space that is more readily resolved by EnKF).

\section{IMPLEMENTATION OF PILOT POINT}

The proposed methodology has been implemented for history matching of a three facies synthetic model within EnKF framework. Facies distribution is the unknown model parameter. The objective is to update the facies indicators by means of integrating observed data while honoring 
conditioning data at wells, maintaining spatial correlation of parameters and the reference probability distributions.

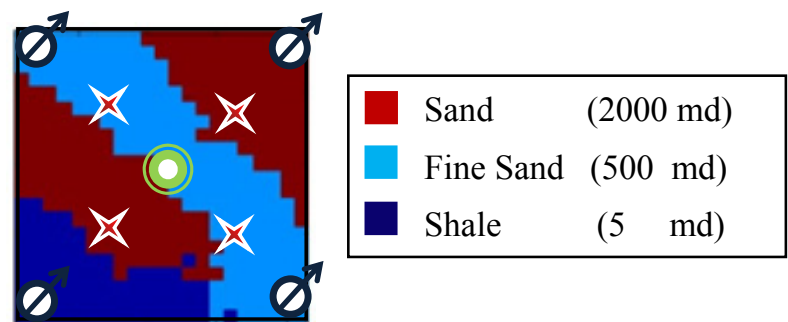

Fig. 1. Facies distribution of Benchmark Case and the well locations ( () Producer, \& Injector, X Pressure Observation).

\subsection{Model Description}

The model was in two dimensional $(21 \times 21$ grid blocks in the $\mathrm{x}-\mathrm{y}$ direction) and three distinct facies namely sand (S), fine sand (FS) and shale were present in the model. The reference facies proportions were $45 \% \mathrm{~S}, 37 \% \mathrm{FS}$, and $18 \%$ shale. Petrophysical properties for each facie is defined based on geologic information collected at all 9 well locations, which include facie classification, porosity, and permeability values. Permeability within each facies was assumed to be uniform with an average value and rock porosity of different facies were assumed to be constant (30\%) throughout the model.

Facies distribution for the "benchmark case" was generated via unconditional SIS, as implemented in the GSLib (Deutsch and Journel 1997). Based on the geological properties of the reservoir obtained from regional studies, an experimental variogram is inferred as an exponential variogram model with maximum and minimum correlation lengths of $1800 \mathrm{ft}$ and $700 \mathrm{ft}$, respectively, along an azimuth angle of $135^{\circ}$.

Table. 1. Synthetic reservoir model parameters

\begin{tabular}{|l|l|}
\hline Model Dimensions & $21 \times 21 \times 1$ grids in X, Y and Z \\
\hline Grid Dimensions & $100 \times 100 \times 20 \mathrm{ft}$ \\
\hline Reservoir Depth & $3000 \mathrm{ft}$ \\
\hline Porosity & $30 \%$ constant \\
\hline Permeability Range & 50,500 and $2000 \mathrm{md}$ \\
\hline Operating bottomhole & Producer: $2300 \mathrm{psi}$ \\
Pressure & Injectors: $2500 \mathrm{psi}$ \\
\hline Initial Reservoir Pressure & 2500 psi \\
\hline Total Generated & 10 years history \\
Observed Data & 23 years prediction \\
\hline
\end{tabular}

Eclipse black-oil simulator was used as the reservoir simulator. Production and injection wells were operated at constant bottomhole pressure. Synthetic field observed data set, which consists of oil production rate, gas oil ratio and water cut of the producer, water injection rates; and bottomhole pressure of the observation wells, was obtained by subjecting the benchmark case to reservoir simulator. These observed data were collected on a monthly basis for 30 years.

\subsection{Base Case}

In this case it was intended to examine the DCT-EnKF method and its performance for updating multiple facies distributions. An initial ensemble of 60 facies distribution was generated via conditional SIS. The facies observations at the well locations, i.e. the producer, injectors and observation wells (9 hard data) were used as the conditioning data. The variogram parameters and the reference distribution were same as the benchmark case. Facies proportions of the simulated realizations were within the range of $5 \%$ of the benchmark case. History matching was performed for 10 update steps. Updates were every year.
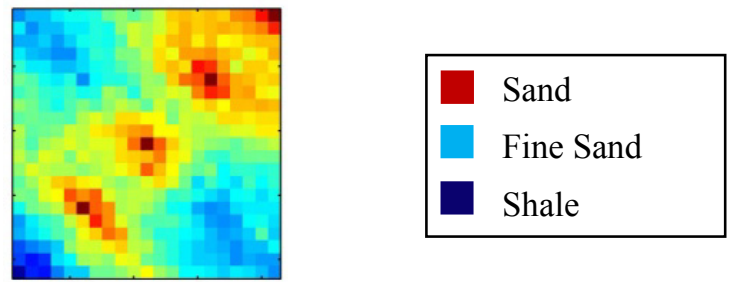

Fig. 2. Mean of initial ensemble of facies indicators for Base Case

In order to evaluate the overall quality of the history match, the commonly used R-square value (Chitralekha et al. 2010, Gul et al. 2011) was implemented to calculate the normalized measure of the fit:

$R_{i, P}^{2}=1-\sum_{t=t_{1}}^{t_{N}}\left(d_{t}^{o b s, P}-\hat{d}_{t}^{i}\right)^{2} / \sum_{t=t_{1}}^{t_{N}}\left(d_{t}^{o b s, P}-\bar{d}^{o b s, P}\right)^{2}$

In equation (4), $R_{i, P}^{2}$ is defined for $i^{\text {th }}$ realization and $p^{\text {th }}$ production variable. $\bar{d}^{o b s, p}$ is the time average of observed data over the $t=t_{l}$ to $t_{N}$ interval. The overall quality of the match for each realization is calculated as:

$$
R_{i}^{2}=\sum_{p=1}^{N_{p r o d}} R_{i, p}^{2} / \omega_{p}
$$

The calculated $\mathrm{R}$-square values represent the mismatch in the predictions as a unit less number. R-square can be calculated for a specific observation (e.g. oil production rate of a specific well) using equation 4 . In case the overall quality of the match for each realization is required, the weighted R-square value (derived from equation 5) can be implemented to account for different observed data having various units and importance level and unite them into a single value. After calculating the R-square value for each realization (either equation 4 or 5 ) the mean of R-square values for different realizations is used to investigate the quality of the match and compare results of different cases.

Figure 3 depicts the average facies map of the ensemble after different update steps. The updated facies distributions are scattered containing short-scale variations which are not geologically realistic and will affect recovery performance. Noise reduction techniques from image processing are used to smooth these variations and match variogram parameters. It is important to note that smoothing techniques are not used to reproduce spatial continuity in model parameters; it is used only to remove some unphysical short-scale discontinuities. 

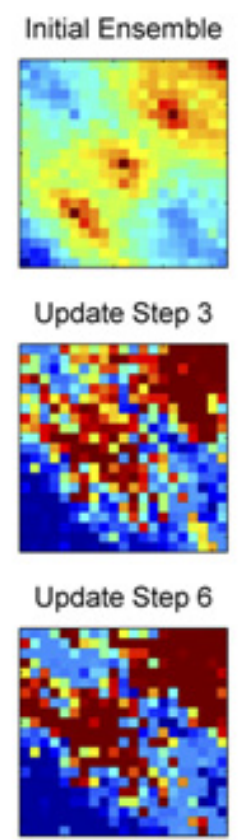

Update Step 9

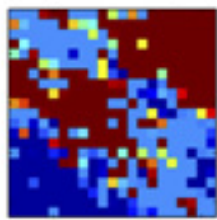

Update Step 6

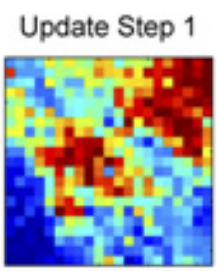

Update Step 4

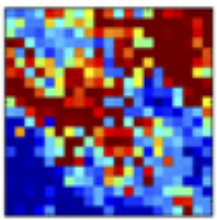

Update Step 7

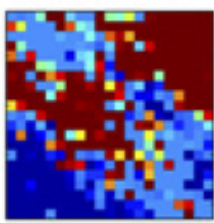

Update Step 10

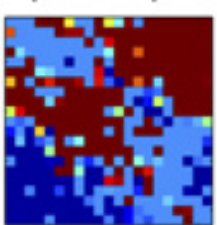

Update Step 2

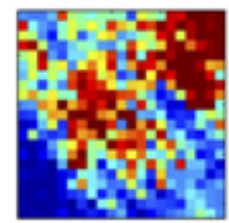

Update Step 5

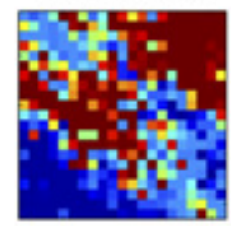

Update Step 8
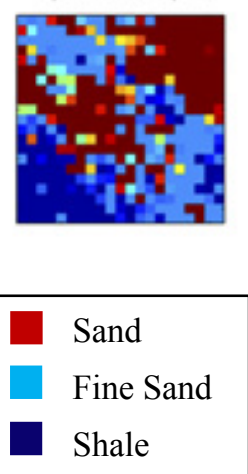

Fig. 3. Average areal map of the facies indicators of all realizations at different update steps, for Base Case.
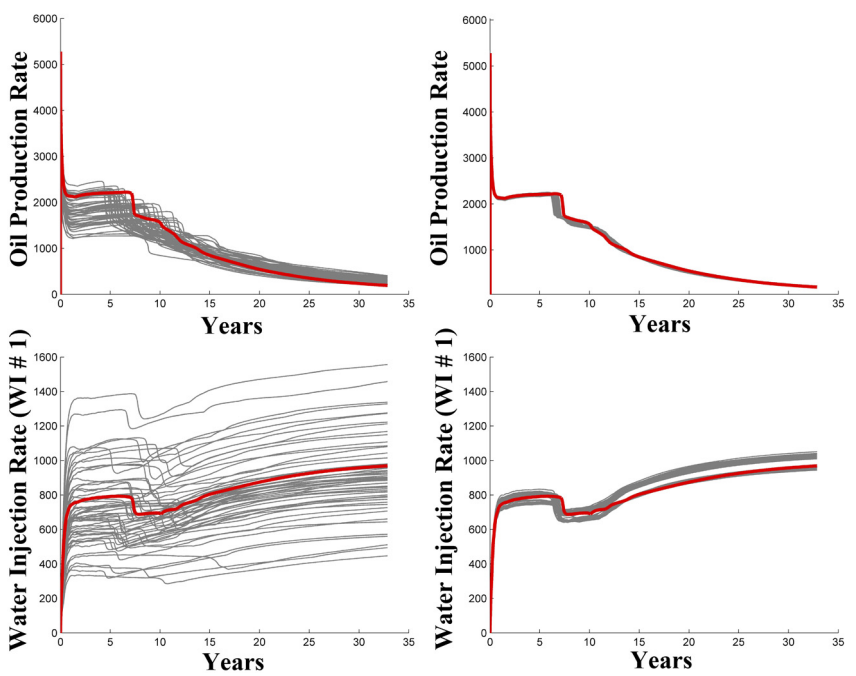

Fig. 4. Oil production rate and water injection rates before (left column) and after (right column) history match for Base Case.

\subsection{Noise reduction using image processing algorithms}

Post-processing or smoothing facies realizations is a promising approach to remove geologically unrealistic shortscale variations. However, the reference distribution should be honored while doing so. Different noise removal algorithms have been introduced in geostatistical modelling such as Maximum a-posteriori selection method - and image processing literature. Low pass, Median and Out-range pixel smoothing are some of the linear and nonlinear techniques that are implemented to remove random noise. Using these approaches would not necessarily maintain facies proportions and for instance facies with relatively small proportions would be poorly preserved.

Maximum a-posteriori selection (MAPS): This technique replaces the facies type at each location by the most probable facies type based on the local neighbourhood. The probability of each facies type, in the local neighbourhood, is based on closeness to the location, whether or not the value is a conditioning datum, and mismatch from the target proportion (Deutsch 1998).

Median Filtering: It is a nonlinear technique which is being used in image processing. Median filtering smoothes the image and is thus useful in removing random noise such as salt and pepper or impulsive noise and meanwhile preserving edges (Lim 1990). It can also preserve discontinuities in a step function and can smooth a few pixels whose values differ significantly from their surroundings without affecting other pixels.

Mode Filtering: This technique is similar to the Median filtering, however, mode of the data is assigned to the target pixel instead of the median value.

We have implemented mode filtering algorithm to smooth the updated realizations, and median filtering for smoothing probability maps; however, other noise reduction techniques can be easily integrated into the proposed formulation. Figure 5(a) shows the facies map of an updated realization with the lowest R-square value. Markedly the realization is noisy, has discontinuity and lots of short scale variations. The purpose of applying smoothing techniques is to remove these unrealistic scattered data and noise. Figure 5(b) shows the same map after applying the mode filter. Obviously this map seems to be more realistic.

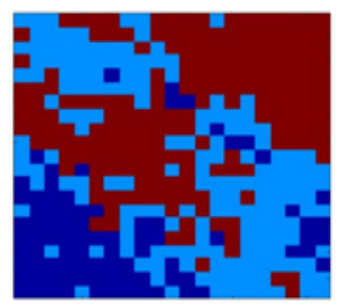

(a)

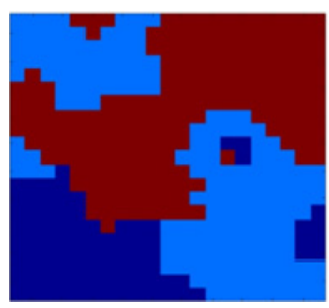

(b)
Fig. 5. Areal map of an updated realization with the lowest R-square value for Base Case before (a) and after (b) smoothing.

Figures 6(a) and 6(b) compares the experimental variogram of the same realization before and after smoothing, and the Benchmark Case. Before removing smoothing the calculated experimental variogram for small separation distances is higher in both azimuths, which are close to the benchmark case after applying the filter. The smoothed experimental variogram data along the major direction of continuity $\left(135^{\circ}\right.$ azimuth), although not perfect, at least are close to the Benchmark Case in the short ranges (i.e. up to $600 \mathrm{ft}$ ). However, the same plot along the minor direction of 
continuity ( $45^{\circ}$ azimuth) shows that the updated realization in Base Case is not acceptable. The slope of the curves is different and the semi-variogram of Base Case does not reveal the cyclic nature which is evident in Benchmark Case. (a)

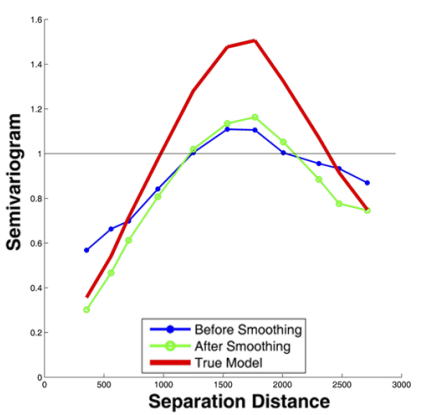

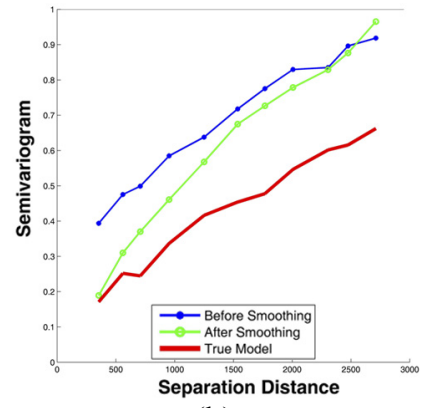

(b)
Fig. 6. Experimental Variogram of an updated realization of Base Case along the horizontal azimuths of 45 (a) and 135 (b) degrees.

\subsection{Regenerated realizations using Base Case final results using Pilot Point method}

In this case study, the DCT-EnKF updated facies realizations obtained at the last update step (20th update) of Base Case are subjected to the proposed Pilot Point scheme. The top realizations with least amount of mismatch are identified as high-weight particles. These realizations are used to calculate the probability density function (pdf) of different facies at different locations. Noise reduction techniques are applied to the probability maps. The smoothed pdf data are further implemented to define facies types at pilot point locations. The detail procedure to generate new set of realizations is described below:

1) The updated realizations are sorted based on the quality of the match (RMSE), as well as the variogram and facies proportions similarity to the geological data of the formation, and the top $20 \%$ of the realizations were selected.

2) Using the selected realizations, probability of the existence of each facies at different grid block locations is calculated.

3) The calculated pdf values contain short scale variations and are noisy. Median noise reduction technique was implemented to remove the short scale variations. Median filter removes the noise and smoothes the edges.

4) After smoothing pdf values, summation of the probability of the facies at a specific grid block location is not necessarily equal to unity. A correction step has been implemented so that the sum of pdf values of different facies at each grid block location would be one.

5) Grid block locations where probability of the occurrence of any facies is above $90 \%$ are specified.

6) A total of 10 percent of the total grid block numbers was intended to be selected as pilot points. Number of pilot points in each facies was calculated based on the initial estimate of the facies proportions.
7) The desired number of pilot points in each facies is randomly selected from specified locations in step (4).

8) New set of realizations are generated via SIS using conditioning hard data of the wells and the pilot points.

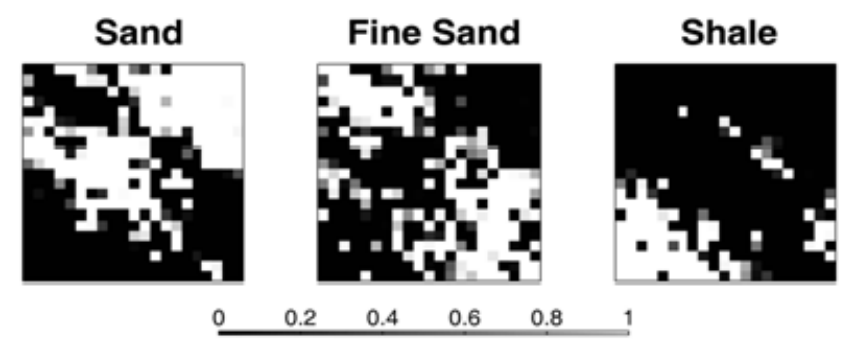

Fig. 7. Calculated pdf values for different facies.

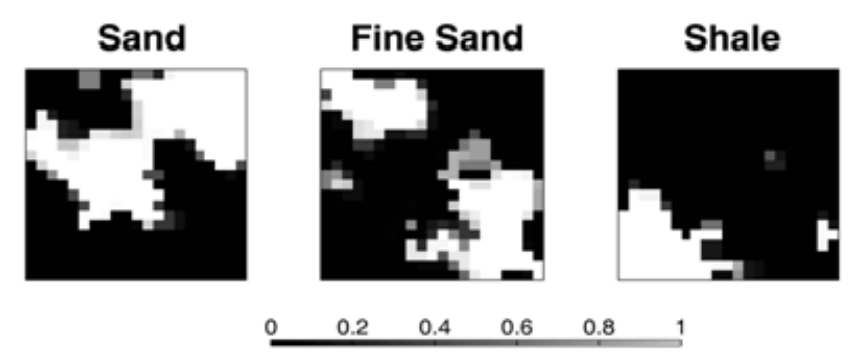

Fig. 8. Smoothed pdf maps.
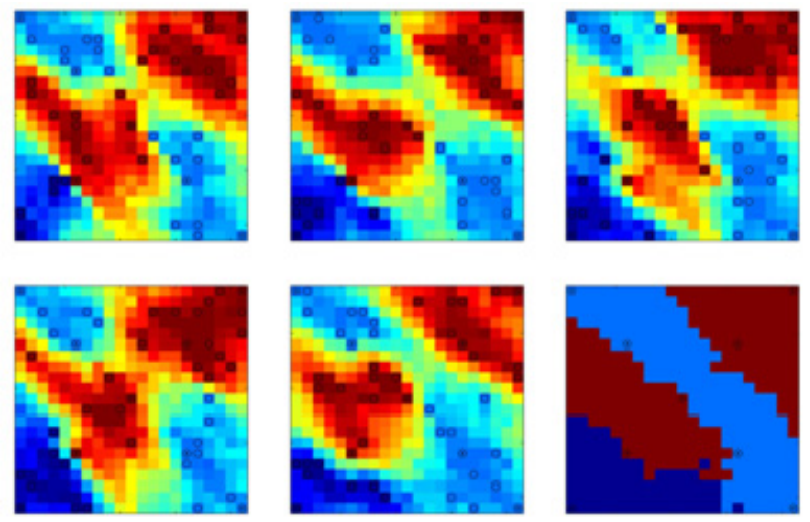

Fig. 9. Areal map of different set of realizations generated using Pilot Point method, compared to the Benchmark Case.

The prescribed technique was used and the last two steps (steps 7 and 8) were repeated five times to generate five ensembles. These ensembles were combined (50 realizations) and were updated from the initial using DCT-EnKF (ten steps, one update per year). Figure 10 depicts the average facies map of the realizations after different update steps. The updated realizations of this case contain short scale variations as well. The results are far better than the Base Case however, implementing smoothing algorithms are helpful. Mode filtering algorithm was used to remove the unrealistic scattered data and noise. Figure 12(a) shows the facies map of an updated realization with the lowest R-square value. The realization is a bit noisy and has a few unrealistic short scale variations. Figure 12(b) shows the same map after applying the mode filter. Figure $13(\mathrm{a}, \mathrm{b})$ depict the calculated experimental semi-variogram plot of the Pilot Point Case compared to the Benchmark. The plots show that before 
smoothing, the calculated experimental semi-variogram values are a bit higher, which is improved after implementing the smoothing technique. The short scale variations are removed and the data closely match the benchmark semivariogram values especially along the $135^{\circ}$ azimuth. The plots clearly reveal the cyclic behaviour of the semivariogram along the minor direction of continuity though not perfectly matching the points.
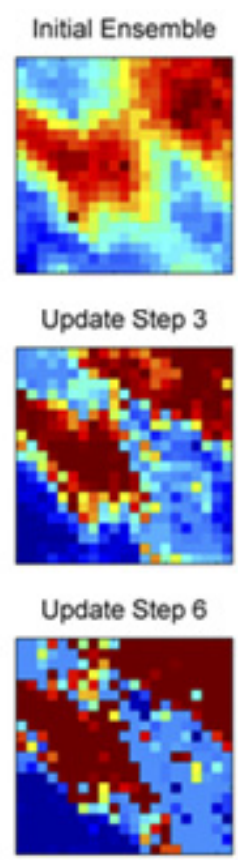

Update Step 9

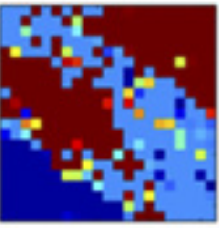

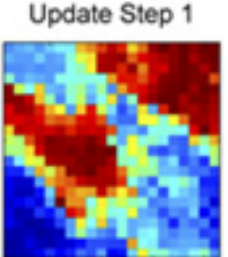

Update Step 4

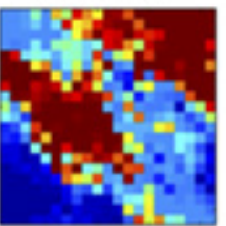

Update Step 7

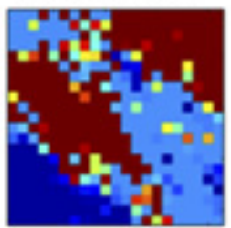

Update Step 10

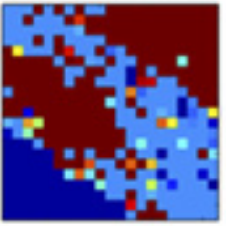

Update Step 2

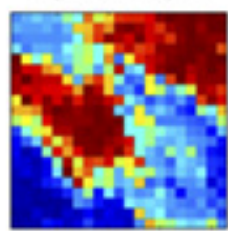

Update Step 5

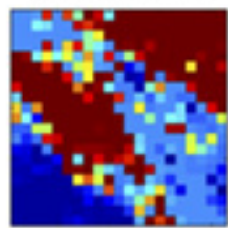

Update Step 8
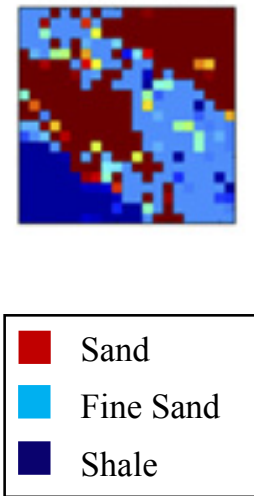

Fig. 10. Average areal map of the facies indicators of all realizations at different update steps. The initial ensemble was re-generated using Pilot Point method.
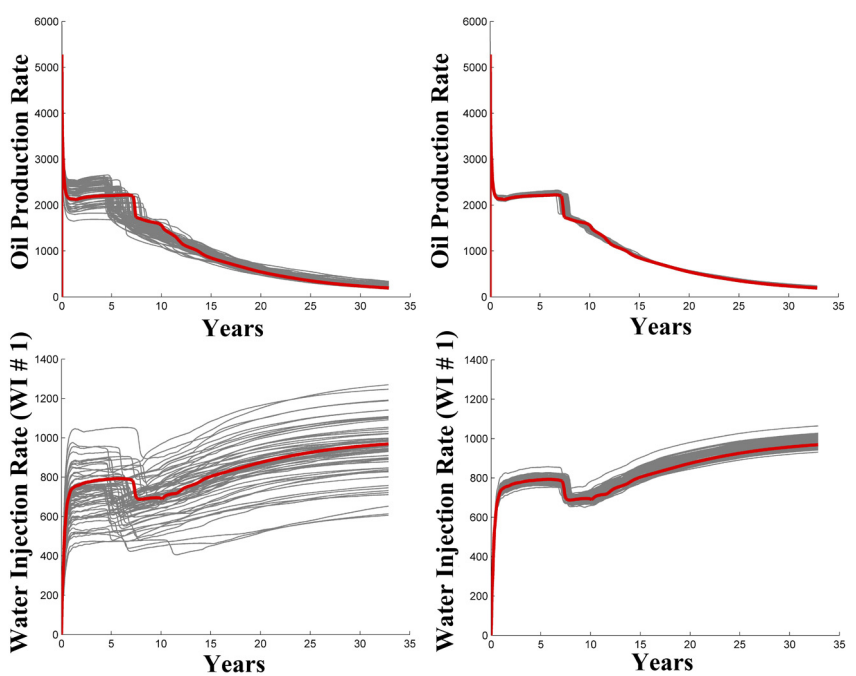

Fig. 11. Oil production rate and water injection rates before (left column) and after (right column) history match for Pilot Point Case.

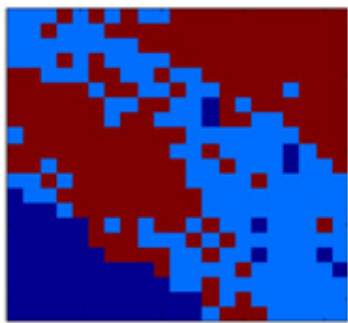

(a)

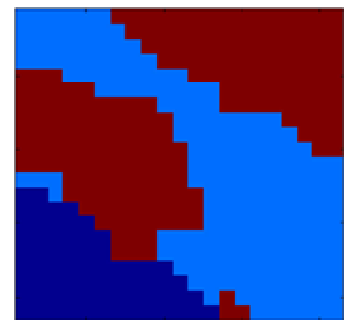

(b)
Fig. 12. Areal map of an updated realization with the lowest R-square value for Pilot Point Case before (a) and after (b) smoothing.

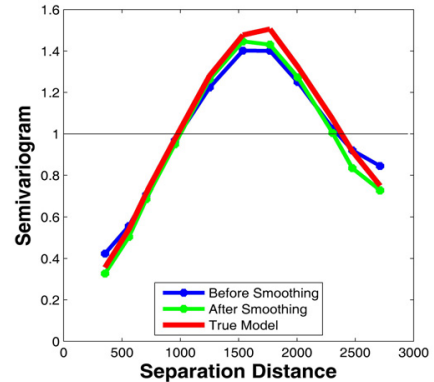

(a)

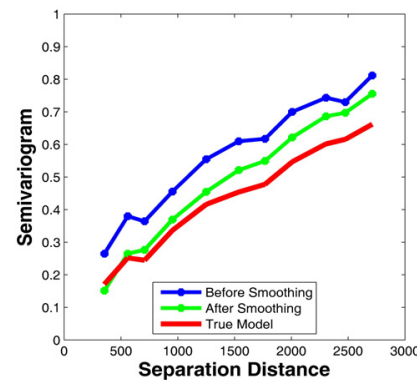

(b)
Fig. 13. Experimental Variogram of an updated realization of Pilot Point Case along the horizontal azimuths of 45 (a) and 135 (b) degrees.

Figure 14 depicts the facies proportions of different cases before and after smoothing. Clearly the facies proportions are not preserved in Base Case. However, for the case with regenerating the realizations using proposed pilot point method, the facies proportions are close to the Benchmark Case. Besides the graph shows that smoothing algorithm has not affected the facies proportions.

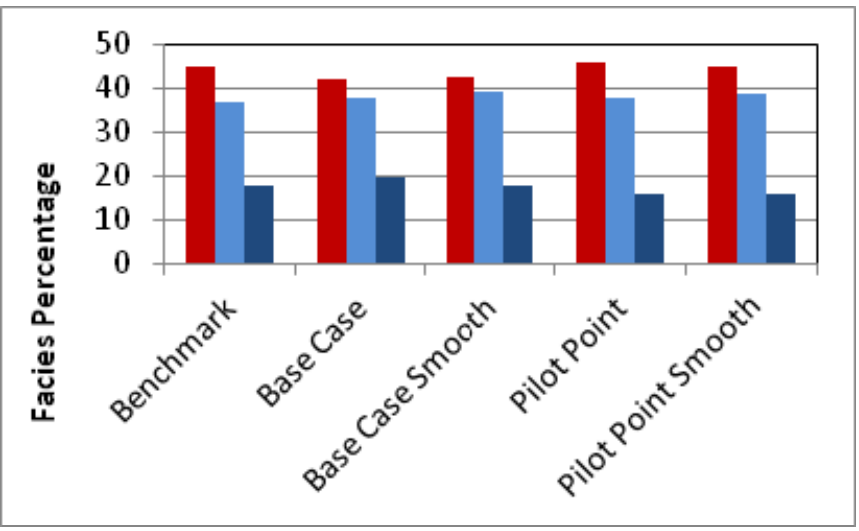

Fig. 14. Facies histogram of different cases, compared to the Benchmark Case.

\section{CONCLUSIONS}

A novel predictor-corrector type bias correction procedure is proposed to (1) correct for the loss of non-Gaussian contributions in model parameters after EnKF update and to (2) honor the reference distribution obtained from static 
geologic information. The method involves combining a new pilot-point formulation with the DCT-EnKF approach. The results on numerical experiments conducted on multi-facies reservoir model showed the success of regenerating new realizations in facies pattern detection. The smoothing algorithm also assisted to correct for the short-scale variability of the distributions and the facies probability maps. This predictor-corrector-analysis type approach outperformed in terms of observed dynamic data are match as well as retaining non-Gaussian geological features such as short scale-variability, reference facies proportions and variogram cyclicity (spatial distribution of model parameters).

\section{ACKNOWLEDGMENT}

The authors wish to acknowledge the financial support from NSERC Discovery Grant and the University of Alberta for this research. ECLIPSE reservoir simulator was provided by Schlumberger.

\section{REFERENCES}

Aanonsen, S.I., Naevdal, G., Oliver, D.S., Reynolds, A.C. and Valles, B., September 2009. The Ensemble Kalman Filter in Reservoir Engineering - a Review. SPE J, Vol. 14 No. 3, 393-412.

Agbalaka, C. and Oliver, D.S. 2008. Application of the EnKF and Localization to Automatic History Matching of Facies Distribution and Production Data. Mathematical Geosciences, Vol. 40 NO. 4, 353-374.

de Marsily, G., 1984. Spatial Variability of Properties in Porous Media: A Stochastic Approach, in Fundamentals of Transport in Porous Media. Proceedings of NATO Advanced Study Institute on Mechanics of Fluids in Porous Media, Bear J. and Corapcioglu M. Y., Martinus Nijhoff, Boston. 719-769.

Deutsch, C.V. 1998. Cleaning categorical variable (lithofacies) realizations with maximum a-posteriori selection. Computaters \& Geosciences. Vol. 24, NO.6, 551-562.

Deutsch, C.V., Journel, A.G., 1997. GSLIB: Geostatistical Software Library and User's Guide. second edition, Oxford University Press, New York.

Dovera, L., Della Rossa, E. 2010. Multimodal Ensemble Kalman Filtering Using Gaussian Mixture Models. Computational Geosciences. Vol. 15, No.2, 307-323.

ECLIPSE 100 (Black Oil), Reference manual and technical description. Schlumberger GeoQuest, 2009.

Evensen, G., 2007. Data Assimilation: The Ensemble Kalman Filter. Springer, Berlin.

Gul, A., Nejadi, S., Shah, S., Trivedi, J., 2011. Make Use of Dynamic Data - A Constrain Based EnKF for SAGD Reservoir Characterization and Production Management. Paper WHOC 11-568 presented at World Heavy Oil Congress held in Edmonton, Alberta, Canada, March 1417.

Jafarpour, B., McLaughlin, D.B. 2008. History Matching with an Ensemble Kalman Filter and Discrete Cosine Parameterization. Computational Geosciences. Vol. 12, 227-244.
Jafarpour, B., Tarrahi, M. 2011. Assessing the Performance of the Ensemble Kalman Filter for Subsurface Flow Data Integration under Variogram Uncertainty. Water Resources Research, Vol. 47, W05537, 26.

Lim, Jae S., Two-Dimensional Signal and Image Processing. Englewood Cliffs, NJ, Prentice Hall, 1990, 469-476.

Liu, N., Oliver, D.S. 2005. Ensemble Kalman Filter for Automatic History Matching of Geologic Facies. Journal of Petroleum Science and Engineering, Vol. 47, 147161.

Lorentzen, R. J., Fjelde, K. K., Froyen, J., Lage, A. C., Naevdal, G. And Vefring, E. H. 2001. Underbalanced and Low-Head Drilling Operations: Real Time Interpretation of Measured Data and Operational Support. Paper SPE 71384 presented at the SPE Annual Technical Conference and Exhibition, New Orleans, September 30- October 3.

Lorentzen, R.J., Nævdal,G. And Shafieirad, A. 2011. Estimating Facies Felds using the Ensemble Kalman Filter and Distance Functions-Applied to Shallowmarine Environments. SPE Paper 143031 presented at the SPE EUROPEC/EAGE Annual Conference and Exhibition, Vienna, Austria, May 23-26.

Lorentzen, R.J., Flornes, K.M., and Nævdal,G. 2012. History Matching Channelized Reservoirs Using the Ensemble Kalman Filter. SPE J. 17 (1): 137-151. SPE-143188-PA.

Moreno, D. and Aanonsen, S.I. 2011. Continuous Facies Updating Using the Ensemble Kalman Filter and the Level Set Method. Mathematical Geosciences. Published Online: 01 July DOI: 10.1007/s11004-0119347-4.

Nejadi, S., Trivedi, J., Leung, J., 2011. Improving Characterization and History Matching Using Entropy Weighted Ensemble Kalman Filter for Non-Gaussian Distributions. SPE Paper 144578 presented at the SPE Western North American Regional Meeting, Anchorage, Alaska, USA, May 7-11.

Nejadi, S., Trivedi, J., Leung, J., 2012. Estimation of Geological Facies Boundaries Using Categorical Indicators with P-Field Simulation and Ensemble Kalman Filter (EnKF). SPE Paper 153645 presented at the SPE Latin American and Caribbean Petroleum Engineering Conference, Mexico City, Mexico, April 16-18.

Rao, K.R. and Yip, P., 1990. Discrete Cosine Transform: Algorithms, Advantages, Applications. Academic Press, Boston.

RamaRao, B.S., LaVenue, A.M., de Marsily, G., Marietta, M.G., 1995. Pilot Point Methodology for Automated Calibration of an Ensemble of Conditionally Simulated Transmissivity Fields 1. Theory and Computational Experiments. Water Resources Research. VOL. 31, NO. 3, 475-493.

Zafari, M. and Reynolds, A.C. 2007. Assessing the Uncertainty in Reservoir Description and Performance Predictions with the Ensemble Kalman Filter. SPE J. Vol. 12, NO. 3, 382-391. SPE-95750-PA 